\title{
Qualidade de vida e equilíbrio de idosas praticantes de hidroginástica de um Programa
} de Atividades Físicas e Esportivas

Quality of life and balance of elderly women practicing water aerobics in a Physical and Sports Activities Program

Calidad de vida y equilibrio de ancianas practicantes de hidrogimnasia de un Programa de Actividades Físicas y Deportivas

Daniele Bueno Godinho Ribeiro André Santos Cunha Silva ${ }^{2}$

Guilherme Luiz Silva Ferreira ${ }^{2}$ Alexandre Lima de Araújo Ribeiro ${ }^{3}$ Gheorges Lucas Parente Rodrigues ${ }^{1}$

\section{Resumo}

Introdução: a hidroginástica propicia melhor qualidade de vida em relação aos benefícios que oferece ao idoso, proporcionando uma vida mais saudável e ativa a essa população. Além disso, a prática regular de atividade física esta diretamente ligada ao equilíbrio. Objetivo: analisar a qualidade de vida e o equilíbrio das idosas participantes de hidroginástica do Programa de Atividades Físicas e Esportivas, da cidade de Gurupi/TO. Metodologia: aplicou-se o Short-Form Health Survey (SF-36) e a Escala de Equilíbrio de Berg (EEB) em 19 idosas $(67,47 \pm 5,66)$ praticantes regulares de hidroginástica no Programa de Atividades Físicas e Esportivas na cidade de Gurupi no Tocantins. Resultados: com exceção dos aspectos emocionais com média de 40,3, os escores encontrado no SF-36 foram considerados satisfatórios nos outros domínios: aspectos físicos $(60,5)$, dor $(64)$, estado geral de saúde $(79,8)$, vitalidade $(62,6)$, aspectos sociais $(77,1)$, saúde mental $(75,7)$. Em relação a EEB obteve-se um excelente resultado $(53,7 \pm$ 2,05). Conclusão: apesar de não possuírem um bom aspecto emocional, as idosas praticantes de hidroginástica no Programa de Atividade Físicas e Esportivas, da cidade de Gurupi/Tocantins/Brasil, possuem uma boa qualidade de vida e um excelente equilíbrio. Palavras-chave: Idosos. Qualidade de Vida. Equilíbrio. Hidroginástica.

\footnotetext{
Abstract

Introduction: hydrogynas provides a better quality of life in relation to the benefits it offers the elderly, providing a healthier and more active life for this population. In addition, the regular practice of physical activity is directly linked to balance. Objective: to analyze the quality of life and the balance of the elderly participants in water aerobics of the Program of Physical and Sports Activities, of the city of Gurupi-TO. Methodology: the Short-Form Health Survey (SF-36) and the Berg Balance Scale (BSE) were applied to 19 elderly women $(67.47 \pm 5.66)$ regular water aerobics practitioners in the Physical and Sports Activities Program at city of Gurupi in Tocantins. Results: with the exception of the emotional aspects with a mean of 40.3, the scores found in the SF-36 were considered satisfactory in other domains: physical aspects (60.5), pain (64), general health status

1. Universidade Federal do Tocantins - UFT, Miracema do Tocantins, Tocantins. 2. Centro Universitário UnirG UnirG, Gurupi, Tocantins. 3. Universidade de Brasília - UnB, Brasília, Distrito Federal. Endereço para correspondência: Daniele Bueno Godinho Ribeiro, Avenida Lourdes Solino, Setor Universitário, Universidade Federal do Tocantins - UFT, Miracema do Tocantins, Tocantins, CEP: 77650-000, danielebueno@uft.edu.br .

Este conteúdo utiliza a Licença Creative Commons Attribution 4.0 International License Open Access. This content is licensed under a Creative Commons attribution-type BY. ISSN 2595 - 0096.
} 
(79.8), vitality (62.6), social aspects (77.1), and mental health (75.7). In relation to BSE, an excellent result was obtained $(53.7 \pm 2.05)$. Conclusion: Although they do not have a good emotional aspect, elderly women practicing water aerobics in the Physical and Sports Activity Program of the city of Gurupi/Tocantins/Brasil,have a good quality of life and an excellent balance.

Keywords: Elderly. Quality of Life. Balance Hydrogynastic.

\section{Resumen}

Introducción: La hidrogimnasia propicia una mejor calidad de vida en relación a los beneficios que oferece al adulto mayor, proporcionando una vida más saludable y activa a esa población. Además, la práctica regular de actividad física está directamente ligada al equilibrio. Objetivo: analizar la calidad de vida y el equilibrio de las adultas mayores participantes de hidrogimnasia del Programa de Actividades Físicas y Deportivas, de la ciudad de Gurupi-TO. Metodología: se aplicó el Short-Form Health Survey (SF-36) y la Escala de Equilibrio de Berg (EEB) en 19 adultas mayores $(67,47 \pm 5,66)$ practicantes regulares de hidrogimnasia en el Programa de Actividades Físicas y Deportivas de la ciudad de Gurupi en Tocantins. Resultados: con excepción de los aspectos emocionales, los puntajes encontrados en el SF-36 se consideraron satisfactorios en los otros ámbitos: aspectos físicos $(60,5)$, dolor $(64)$, estado general de salud $(79,8)$, vitalidad $(62,6)$, aspectos sociales $(77,1)$, salud mental $(75,7)$. En cuanto a la EEB se obtuvo un excelente resultado $(53,7 \pm 2,05)$. Conclusión: a pesar de no tener un buen aspecto emocional, las adultas mayores practicantes de hidrogimnasia en el Programa de Actividad Físicas y Deportivas, de la ciudad de Gurupi/Tocantins/Brasil, poseen una buena calidad de vida, y un excelente equilibrio.

Palabras clave: Adultas mayores. Calidad de vida. SF-36. Equilibrio. Hidrogimnasia.

\section{INTRODUÇÃO}

Como está a qualidade de vida e o equilíbrio dos idosos? Nos últimos anos observa-se uma elevada taxa de crescimento da população idosa no Brasil e no mundo. Segundo o Instituto Brasileiro de Geografia e Estatística (IBGE), o número de idosos, no Brasil, com 60 anos em 2012 era de 25,4 milhões e em 2017 superou a marca dos 30,2 milhões ${ }^{1}$.

\section{A World Health Organization}

(WHO) define como qualidade de vida “a percepção do indivíduo de sua posição na vida no contexto da cultura e sistema de valores nos quais eles vivem em relação aos seus objetivos, expectativas, padrões e preocupações”2.
Contudo, quando se discute sobre a qualidade de vida nesta população, entende-se que o idoso com uma adequada qualidade de vida é aquele que possui uma boa condição física, funcional, social e emocional ${ }^{3}$. Por isso, a qualidade de vida pode estar diretamente ligada a diversos fatores, como a (a) prática regular de atividades físicas, seja esta recreacional, de rendimento ou educacional; (b) capacidade de realizar tarefas do cotidiano sem auxílio de terceiros; (c) relacionamentos; (d) saúde mental e o bem-estar psicológico.

$$
\text { Dentre essas variáveis }
$$
relacionadas à qualidade de vida, a 
prática de atividade física voltada para o equilíbrio pode ser importantíssimo, uma vez que por possuir uma integração de vários processos, dentre eles a força muscular e o tempo de reação, um bom equilíbrio é capaz de proporcionar uma maior autonomia ao idoso ${ }^{4}$. Além de tornar-se um fator protetivo para o mesmo, uma vez que quanto maior o domínio e capacidade de equilíbrio deste, menor a chance de ocorrências de quedas, que é um dos fatores que têm causado maior número de mortalidade e incapacidade nesta população ${ }^{4}$.

Para Sova $1998^{5}$ a hidroginástica propicia melhor qualidade de vida em relação aos benefícios que ela oferece ao idoso, como melhora na força, equilíbrio e mobilidade articular, propiciando uma vida mais saudável. Além disso, o exercício aquático ocupa destaque em atividades propostas por academias e clubes para esta população. Portanto, o objetivo deste estudo é analisar a qualidade de vida e o equilíbrio das idosas participantes de hidroginástica do Programa de Atividades Físicas e Esportivas, da cidade de Gurupi/Tocantins/Brasil.

\section{MÉTODOS}

Esta pesquisa caracteriza-se como quantitativa descritiva. Foi aprovada pelo Comitê de Ética e Pesquisa com Seres Humanos do Centro Universitário
UnirG (parecer $n^{\circ}$. 985977) e realizada no Programa de Atividades Físicas e Esportivas (PROAFE), desenvolvido no município de Gurupi/Tocantins/Brasil.

\section{Participantes}

Como critério de inclusão as idosas deveriam ter entre 60 e 80 anos, praticarem exercício físico sistematizado e possur assiduidade mínima de 6 meses no PROAFE. Foram excluídas as idosas que possuíam alguma (a) doença ou dor musculoesquelética, (b) doença neurológica, como Parkinson, demência ou acidente vascular cerebral, (c) deficiência visual, ou (d) doença cardiovascular que prejudicasse $\mathrm{o}$ exercício físico.

Medidas do Estudo

Devido a fácil aplicação e entendimento por partes dos voluntários, além do baixo custo e mínimo risco em sua realização, optouse por utilizar o Short-Form Health Survey (SF-36) e a Escala de Equilíbrio de Berg (EEB), que avaliam qualidade de vida e equilíbrio, respectivamente.

\section{O Short-Form Health Survey} (SF-36) é um instrumento validado ${ }^{6}$ de fácil administração, e compreensão, que analisa a qualidade de vida, por meio de 11 questões de múltipla escolha. Consiste em um questionário multidimensional que avalia a capacidade funcional, limitação dos 
aspectos físicos, dor, estado geral da saúde, vitalidade, aspectos sociais e emocionais, além de saúde mental. Essa avaliação retorna um escore final de 0 à 100 pontos, onde o 0 corresponde ao pior e o 100 ao melhor resultado.

A Escala de Equilíbrio de Berg é uma avaliação funcional do equilíbrio do idoso, e baseia-se em 14 atividades comuns do dia a dia, com uma pontuação que varia entre 0 e 56 pontos. É um instrumento de fácil aplicação, validado e adaptado para a língua portuguesa (Tabela 1) que avalia o controle postural através de diferentes exigências de força, equilíbrio dinâmico e flexibilidade ${ }^{7.9}$.

\section{Procedimentos}

Em um primeiro momento, as voluntárias foram informadas e esclarecidas a respeito da pesquisa e todos os procedimentos a serem realizados, após aceitação para participar da pesquisa, assinou-se o Termo de Consentimento Livre e Esclarecido (TCLE). Na sequência foram aplicados os instrumentos de coleta de dados na seguinte ordem: SF-36 e EEB.

\section{Análise Estatística}

A média e o desvio padrão foram utilizados para descrever os resultados do SF-36 e da EEB. A normalidade foi verificada por meio do teste de Shapiro Wilk, atestada esta, aplicou-se o teste $t$ - student. As análises foram realizadas utilizando o software Statiscal Package for Social Sciences para Windows (SPSS versão 18,0, Chicago IL, EUA) com nível de significância de $\mathrm{p}<0,05$.

\section{RESULTADOS}

Participaram deste estudo 19 idosas (67.47 \pm 5.66 anos) praticantes de hidroginástica.

Observamos que a média dos domínios avaliados do SF 36 obtiveram valores entre 60 a 75 (escala de 0 a 100), o domínio abaixo desse valor foi por aspectos emocionais, todos os outros domínios tiveram valores significativos (tabela 2). A Escala de Berg, demostrou a média e desvio padrão, onde o índice de queda nas idosas analisadas é mínimo, com média 53,7 (escore próximo de 0 , maior chance de quedas, e quanto mais próximo de 56, menor chances de queda) com variável de $\mathrm{p}<0,01$ (tabela 3).

Conforme a Tabela 2, nos domínios avaliados pelo SF-36 observouse, no geral, uma média de 66,87 pontos obtidos, sendo que o menor valor encontrado foi referente ao aspecto emocional (40,3 $\pm 40,9)$. Quanto aos resultados da EEB, constatou-se um risco mínimo de quedas das idosas participantes do estudo $(53,7 \pm 2,05)$.

Tabela 2 - Valores de média e desvio padrão dos domínios avaliados do SF 36 e Escala de Berg 


\begin{tabular}{|c|c|c|c|}
\hline Variáveis & Média \pm DP & Máx.* & Mín. * \\
\hline \multicolumn{4}{|c|}{ Short-Form Health Survey } \\
\hline $\begin{array}{l}\text { Capacidade } \\
\text { Funcional * }\end{array}$ & $\begin{array}{ll}75,0 & \pm \\
17,55 & \end{array}$ & 95 & 40 \\
\hline Aspectos Físicos * & $60,5 \pm 36,6$ & 100 & 00 \\
\hline Dor * & $64,0 \pm 26,2$ & 100 & 12 \\
\hline $\begin{array}{l}\text { Estado Geral de } \\
\text { Saúde * }\end{array}$ & $79,8 \pm 14,2$ & 95 & 45 \\
\hline Vitalidade * & $62,6 \pm 14,1$ & 95 & 35 \\
\hline Aspectos Sociais * & $77,1 \pm 20,6$ & 100 & 40 \\
\hline $\begin{array}{l}\text { Aspectos } \\
\text { Emocionais }\end{array}$ & $40,3 \pm 40,9$ & 100 & 00 \\
\hline Saúde Mental * & $75,7 \pm 15,8$ & 96 & 44 \\
\hline
\end{tabular}

Escala de Equilíbrio de Berg

\begin{tabular}{llll}
\hline $\mathrm{EEB}^{*}$ & $53,7 \pm 2,05$ & 56 & 50 \\
\hline
\end{tabular}

* $\mathrm{p}<0,01$ no Teste $t$ de student. Máx. = máximo; Mín. = mínimo; DP = desvio - padrão.

Fonte: elaboração dos autores.

\section{DISCUSSÃO}

O objetivo do estudo foi analisar a qualidade de vida e o equilíbrio das idosas participantes de hidroginástica do Programa de Atividades Físicas e Esportivas, da cidade de Gurupi/Tocantins/Brasil.

Com exceção dos aspectos emocionais, constatou-se altos escores avaliados pelo SF-36, consequentemente uma boa qualidade de vida, de acordo com a tabela 2 (anexo). Este resultado corrobora com estudos ${ }^{10.13}$ que avaliaram a qualidade de vida de praticantes de atividade física regular. No entanto, chama atenção o baixo valor dos aspectos emocionais, o que sugere que mesmo possuindo autonomia física, funcional e social, os aspectos emocionais podem estar ligados ao ambiente familiar e/ou cotidiano, no caso de idosos institucionalizados. Nesta linha, Caporicci et. al. ${ }^{14}$ comparou a qualidade de vida e o equilíbrio em idosos institucionalizados e não institucionalizados, e obteve como resultado valores abaixo do normal para aspectos emocionais em idosos institucionalizados.

Com relação ao equilíbrio, analisado pela EEB, o resultado encontrado é considerado significativo, uma vez que quanto maior o resultado, ou seja, mais próximo de 56 pontos, menor a chance da ocorrência de um evento de queda, e quanto menor o resultado, maior a chance da ocorrência de um evento de queda. Estes dados corroboram com o estudo conduzido por Pimentel e Scheicher ${ }^{15}$, onde demonstram que os idosos praticantes de atividade física possuem um maior valor (53,46 pontos) no teste, quando comparados com idosos não praticantes de atividade física (47 pontos). Portanto, idosos praticantes de atividade física regular têm menor chance de cair.

Destacamos enquanto ponto forte do estudo a validade interna, visto que as participantes eram frequentadoras do programa de atividades e conseguimos acompanhar controlando as variáveis, porém percebemos a limitação da pesquisa ser realizada 
apenas com participantes do sexo feminino, o que restringe o alcance a população idosa masculina, que em menor quantidade estaria participando de tais práticas esportivas durante a realização da pesquisa.

\section{CONCLUSÃO}

Após tratamento estatístico e análise, podemos concluir de acordo com os objetivos elencados que em relação à prática do exercício físico de hidroginástica realizado pelas idosas pesquisadas, pode-se afirmar como a literatura já prevê, que esta prática favorece a ao qualidade de vida $\mathrm{e}$ equilíbrio, progredindo para uma manutenção ou melhora do estado de saúde de seus praticantes. Porém a discussão de saúde vai além do âmbito da saúde fisiológica e funcional, e percebemos dessa forma que através de dados obtidos e supracitados, ainda assim, se torna necessário uma maior atenção aos idosos em relação aos aspectos emocionais.

Sugere-se que sejam realizados novos estudos comparando idosos, de ambos os sexos, que praticam outros tipos de exercícios físicos e também uma possibilidade de comparar idosos praticantes e não praticantes, como também realizar esta pesquisa com enfoque nas dimensões.

\section{REFERÊNCIAS}

1. INSTITUTO BRASILEIRO DE GEOGRAFIA E ESTATÍSTICA.

Proporção de idosos de 60 anos ou mais e de 65 anos ou mais de idade, Brasil, 1999/2009. Disponível em: http://teen.ibge.gov.br/mao-na-

roda/idosos

2. WORLD

HEALTH

OrGanization. Active Ageing. A Contribution of the World Health Organization to the second United Nations World Assembly on Aging. A Police Framework, Madrid, Spain. April, 2017.

3. PIMENTA, Fausto Aloísio Pedrosa et al. Avaliação da qualidade de vida de aposentados com a utilização do questionário SF-36. 2008.

4. Silva A, Almeida GJM, Cassilhas RC, Cohen M, Peccin MS, Tufik S et al. Equilíbrio, coordenação e agilidade de idosos submetidos à pratica de exercícios físicos resistidos. Revista Brasileira Medicina Esporte, São Paulo, Mar/Abr, 2008; v.14, n.2.

5. Sova R. Hidroginástica na terceira idade. Manole, São Paulo, 1998. 6. Ciconelli RM, Ferra, MB, Santos W, Meinão I, Quaresma MR. Tradução para a língua portuguesa e validação do questionário genérico de avaliação de qualidade de vida SF-36 (Brasil SF-36). Rev Bras Reumatol.1999;39(3):143-50

7. Miyamoto ST, Lombardi Junior I, Berg KO Ramos LR, Natour. J. Brazilian version of the Berg balance scale. Braz J Med Biol Res 2004; 37(9):1411-21

8. Berg K, Wood-Dauphinée S, Williams JI. Measuring balance in the elderly: preliminary development of an instrument. Physiotherapy Canada 1989; 41: 304-11

9. Berg K, Maki B, Williams J. Clinical and laboratory measures of postural balance in an elderly population. Arch Phys Med Rehabil 1992; 73: 1073-80.

10. PIMENTA, Fausto Aloísio Pedrosa et al. Avaliação da qualidade de 
vida de aposentados com a utilização do questionário SF-36. 2008.

11. MOTA, Jorge et al. Atividade física e qualidade de vida associada à saúde em idosos participantes e não participantes em programas regulares de atividade física. Revista brasileira de educação fisica e esporte, v. 20, n. 3, p. 219-225, 2006

12. Acree LS, Longfors J, Fjeldstad AS, et al.: Physical activity is related to quality of life in older adults. Health and Quality of Life Outcomes 4 (37): 1-6, 2006.

13. Binder EF, Schechtman KB, Ehsani AA, et al.: Effects of exercise training on frailty in community-dwelling older adults: results of a randomized, controlled trial. JAGS 50 (12): 1921-8, 2002 .

14. Caporicci S, Neto MFO. Estudo comparativo de idosos ativos e inativos através da avaliação das atividades da vida diária e medição da qualidade de vida; Motricidade. 2011;7(2);15-24.

15. Pimentel RM, Scheicher ME. Comparação do risco de queda em idosos sedentários e ativos por meio da escala de equilíbrio de Berg. Fisioterapia e Pesquisa, São Paulo, janeiro/fevereiro, 2009; v.16, n.1, p.6-10.
Informações do artigo / Information of the article:

Recebido: 21/07/2018

Aprovado: 11/11/2018

Publicado: 31/11/2018

Received: 21/07/2018

Approved: 11/11/2018

Published: 31/11/2018

Mrs. Daniele Bueno Godinho Ribeiro

ORCID: 0000-(0002-9803-3446

Contribuição dos autores

Daniele Bueno Godinho Ribeiro Responsável pela orientação do artigo, análise dos dados e revisão geral;

André Santos Cunha Silva e Guilherme Luiz Silva Ferreira - Responsáveis pela escrita do artigo, coleta dos dados, formatação;

Alexandre Lima de Araújo Ribeiro Responsável pela escrita e revisão geral; Gheorges Lucas Parente Rodrigues Responsável pela revisão de gramática, formatação.

Conflito de interesses

Os autores declaram não haver conflito de interesses.

Financiamento: $\mathrm{O}$ estudo não contou com nenhum tipo de financiamento.

Como citar este artigo:

Ribeiro, D. B. G.; Silva, A. S. C.; Ferreira, G. L. S. e cols. Qualidade de vida e equilíbrio de idosas praticantes de hidroginástica de um Programa de Atividades Físicas e Esportivas. Arq. Bras. Ed. Fís., Tocantinópolis, v. 1, n1. Jan./Jul., p. 167 - 175, 2018. 
Tabelas e/ou Figuras

Tabela 1 - Escala de Equilíbrio de Berg

1) Posição sentada para posição em (4) capaz de levantar-se sem utilizar as mãos e estabilizar-se pé independentemente

Instrução: Por favor, levante-se. (3) capaz de levantar-se independentemente utilizando as mãos Tente não usar suas mãos para se (2) capaz de levantar-se utilizando as mãos após diversas tentativas apoiar

(1) necessita de ajuda mínima para levantar-se ou estabilizar-se

(0) necessita de ajuda moderada ou máxima para levantar-se

2) Permanecer em pé sem apoio

(4) capaz de permanecer em pé com segurança por 2 minutos Instrução: Por favor, fique em pé por (3) capaz de permanecer em pé por 2 minutos com supervisão 2 minutos sem se apoiar.

(2) capaz de permanecer em pé por 30 segundos sem apoio

Se o paciente for capaz de(1) necessita de várias tentativas para permanecer em pé por 30 permanecer em pé por 2 minutos segundos sem apoio sem apoio, dê o número total de (0) incapaz de permanecer em pé por 30 segundos sem apoio pontos o item $N^{o}$ 3. Continue com o item $N^{\circ} 4$.

3) Permanecer sentado sem apoio (4) capaz de permanecer sentado com segurança e com firmeza por 2 nas costas, mas com os pés apoiados minutos

no chão ou num banquinho $\quad$ (3) capaz de permanecer sentado por 2 minutos sob supervisão

Instrução: Por favor, fique sentado (2) capaz de permanecer sentado por 30 segundos sem apoiar as costas com os braços (1) capaz de permanecer sentado por 10 segundos cruzados por 2 minutos.

(0) incapaz de permanecer sentado sem apoio durante 10 segundos

4) Posição em pé para posição (4) senta-se com segurança com uso mínimo das mãos

sentada $\quad$ (3) controla a descida utilizando as mãos

Instrução: Por favor, sente-se. $\quad$ (2) utiliza a parte posterior das pernas contra a cadeira para controlar a descida

(1) senta-se independentemente, mas tem descida sem controle

(0) necessita de ajuda para sentar-se

5) Transferências

(4) capaz de transferir-se com segurança com uso mínimo das mãos

Instrução: Arrume as cadeiras (3) capaz de transferir-se com segurança com o uso das mãos perpendicularmente ou uma de (2) capaz de transferir-se seguindo orientações verbais e/ou frente para a outra para uma supervisão

transferência em pivô. Peça ao (1) necessita de uma pessoa para ajudar

paciente para transferir-se de uma (0) necessita de duas pessoas para ajudar ou supervisionar para cadeira com apoio de braço para realizar a tarefa com segurança

uma cadeira sem apoio de braço, e

vice-versa

6) Permanecer em pé sem apoio (4) capaz de permanecer em pé por 10 segundos com segurança com os olhos fechados $\quad$ (3) capaz de permanecer em pé por 10 segundos com supervisão Instrução: Por favor, fique em pé e (2) capaz de permanecer em pé por 3 segundos feche os olhos por 10 segundos.

(1) incapaz de permanecer com os olhos fechados durante 3 segundos, mas mantém-se em pé

(0) necessita de ajuda para não cair

7) Permanecer em pé sem apoio (4) capaz de posicionar os pés juntos independentemente e com os pés juntos permanecer por 1 minuto com segurança

Instrução: Junte seus pés e fique em (3) capaz de posicionar os pés juntos independentemente e pé sem se apoiar.

permanecer por 1 minuto com supervisão

(2) capaz de posicionar os pés juntos independentemente e permanecer por 30 segundos

(1) necessita de ajuda para posicionar-se, mas é capaz de permanecer com os pés juntos durante 15 segundos

(0) necessita de ajuda para posicionar-se e é incapaz de permanecer nessa posição por 15 segundos

8) Alcançar a frente com o braço (4) pode avançar a frente $>25 \mathrm{~cm}$ com segurança

estendido permanecendo em pé (3) pode avançar a frente $>12,5 \mathrm{~cm}$ com segurança

Instrução: Levante o braço a $90^{\circ}$. (2) pode avançar a frente $>5 \mathrm{~cm}$ com segurança

Estique os dedos e tente alcançar a (1) pode avançar a frente, mas necessita de supervisão

frente o mais longe possível.

(0) perde o equilíbrio na tentativa, ou necessita de apoio externo 
10) Virar-se e olhar para trás por (4) olha para trás de ambos os lados com uma boa distribuição do cima dos ombros direito e esquerdo peso

enquanto permanece em pé $\quad$ (3) olha para trás somente de um lado, o lado contrário demonstra Instrução: Vire-se para olhar menor distribuição do peso

diretamente atrás de você por cima (2) vira somente para os lados, mas mantém o equilíbrio

do seu ombro esquerdo sem tirar os (1) necessita de supervisão para virar

pés do chão. Faça o mesmo por cima (0) necessita de ajuda para não perder o equilíbrio ou cair do ombro direito.

11) Girar 360 graus $\quad$ (4) capaz de girar 360 graus com segurança em 4 segundos ou menos Instrução: Gire-se completamente ao (3) capaz de girar 360 graus com segurança somente para um lado redor de si mesmo.

em 4 segundos ou menos

Pausa. Gire-se completamente ao (2) capaz de girar 360 graus com segurança, mas lentamente redor de si mesmo em sentido (1) necessita de supervisão próxima ou orientações verbais
contrário.
(0) necessita de ajuda enquanto gira

12) Posicionar os pés alternadamente (4) capaz de permanecer em pé independentemente e com no degrau ou banquinho enquanto segurança, completando 8 movimentos em 20 segundos permanece em pé sem apoio Instrução: Toque cada pé movimentos em $>20$ segundos alternadamente no (2) capaz de completar 4 movimentos sem ajuda degrau/banquinho. Continue até que (1) capaz de completar $>2$ movimentos com o mínimo de ajuda cada pé tenha tocado o degrau / (0) incapaz de tentar, ou necessita de ajuda para não cair banquinho quatro vezes.

13) Permanecer em pé sem apoio (4) capaz de colocar um pé imediatamente à frente do outro, com um pé à frente independentemente, e permanecer por 30 segundos

Instrução: Coloque um pé (3) capaz de colocar um pé um pouco mais à frente do outro e diretamente à frente do outro na levemente para o lado, independentemente, e permanecer por 30 mesma linha, se você achar que não segundos

irá conseguir, coloque o pé um (2) capaz de dar um pequeno passo, independentemente, e pouco mais à frente do outro pé e permanecer por 30 segundos

levemente para o lado.

(1) necessita de ajuda para dar o passo, porém permanece por 15 segundos

(0) perde o equilíbrio ao tentar dar um passo ou ficar de pé

14) Permanecer em pé sobre uma (4) capaz de levantar uma perna independentemente e permanecer perna por $>10$ segundos

Instrução: Fique em pé sobre uma (3) capaz de levantar uma perna independentemente e permanecer perna o máximo que você puder sem por 5-10 segundos

se segurar.

(2) capaz de levantar uma perna independentemente e permanecer por $\geq 3$ segundos

(1) tenta levantar uma perna, mas é incapaz de permanecer por 3 segundos, embora permaneça em pé independentemente

(0) incapaz de tentar, ou necessita de ajuda para não cair

Escore Total $/ 56$

Fonte: Berg, 1992. Originalmente publicado em "Berg K, Maki B, Williams J. Clinical and laboratory measures of postural balance in an elderly population. Arch Phys Med Rehabil 1992; 73: 1073-80". 